Layer formation and morphology of ultrasonic spray coated polystyrene nanoparticle layers

Peer-reviewed author version

STRYCKERS, Jeroen; D'OLIESLAEGER, Lien; SILVANO, Joao; Apolinario, C. K.; Laranjeiro, A. C. G.; Gruber, J.; D'HAEN, Jan; MANCA, Jean; ETHIRAJAN, Anitha \& DEFERME, Wim (2016) Layer formation and morphology of ultrasonic spray coated polystyrene nanoparticle layers. In: PHYSICA STATUS SOLIDI A-APPLICATIONS AND MATERIALS SCIENCE, 213(6), p. 1441-1446.

DOI: $10.1002 /$ pssa.201533026

Handle: http://hdl.handle.net/1942/22569 


\title{
Layer formation and morphology of ultrasonic spray coated polystyrene nanoparticle layers
}

\author{
Jan D'Haen ${ }^{1,2}$, J. Manca ${ }^{4}$, A. Ethirajan ${ }^{1,2}$ and, W. Deferme ${ }^{\star 1,3}$ \\ ${ }^{1}$ Institute for Materials Research (IMO-IMOMEC), Hasselt University, Diepenbeek, Belgium \\ ${ }^{2}$ IMEC vzw - Division IMOMEC, Wetenschapspark 1, B-3590 Diepenbeek, Belgium \\ ${ }^{3}$ Flanders Make vzw, Oude Diestersebaan 133, B-3920 Lommel, Belgium \\ ${ }^{3}$ Escola Politecnica, Universidade de Sao Paulo, 05508-010 S. Paulo, SP, Brazil. \\ ${ }^{4} \mathrm{X}-\mathrm{LaB}$, Hasselt University, Diepenbeek, Belgium \\ ${ }^{5}$ Instituto de Quimica, Universidade de Sao Paulo, 05508-000 S.Paulo, SP, Brazil.
}

J. Stryckers ${ }^{1,2}$, L. D'Olieslaeger ${ }^{1,2}$, J.V.M. Silvano ${ }^{3}$, C. K. Apolinrio ${ }^{3}$, A. C. G. Laranjeiro ${ }^{3}$, J. Gruber ${ }^{5}$,

Received 23 August 2007, revised 19 January 2010, accepted ZZZ

Published online ZZZ

Keywords ultrasonic spray coating, nanoparticles, mini-emulsion, thin films, polystyrene NP

Nanoparticles have been increasingly studied due to their unique properties. The deposition of uniform layers with these nanoparticles however is a crucial step in many of their applications. In this work, ultrasonic spray coating is the technique of choice as it is by design well-suited for the deposition of nano-suspension dispersions. The influence of different deposition parameters, ink composition and post-treatment are subject of study in this paper. It is shown in this work that (mass-) production of uniform nanoparticle layers is possible due to the narrow distribution of the droplet size and the deagglomeration of the particles during ultrasonic spray coating. It is also demonstrated that with ultrasonic spray coating it is possible to deposit an eco-friendly, water-based nanoparticle ink into layers with desired thickness and morphology.

1 Introduction Polymeric nanoparticles with controlled surface compositions or functionalization have been increasingly studied and used in a variety of applications, including separation media, adhesive technology, coating, drug delivery systems [1], medical diagnostics[2], etc. Nanoparticle-layers have several advantages over precursor or solution processed layers. The fact that one can use a lower post-treatment temperature enables the deposition on heatsensitive substrates[3][4]. The control over the dimensions and functionalisation of the nanoparticles allows, for instance, engineering the optimal distances of donor and acceptor materials for optimal charge transfer and efficiency in polymer solar cells[5][6]. NP-inks can also be formulated without harmful solvents since there is no need to dissolve the polymer in the ink.[7]

There are various techniques known to deposit thin films of nanoparticles onto a substrate, where the choice depends, besides the desired thickness and morphology of the final layer, on the size of the area that needs to be covered. Drop-casting [8] and spin coating [9] are obviously not suited for large areas deposition. Langmuir-Blodgett deposition [10] offers a high level of control of the particle deposition process and allows for uniform monolayer films but faces similar limitations when it comes to upscaling. Other techniques involve the surface functionalization of substrate and particle before deposition. The drawbacks of these techniques are the required surface modification of the substrate and the limited particle packing density potential.

Dip-coating is a simple, straightforward process and because of this also a widely used coating technique, even being roll to roll compatible. Both for polymer [11] as for metal nanoparticles [12], dip-coating has a range of applications and the lowest process cost for high-volume applications. The large amount of excess material needed and 
the high difficulty to mask areas makes it however less suited for some applications.

Alternatively, spray coating techniques have a great potential for large scale production, since they have virtually no limitation in substrate size and a low utilization of material. It is possible to apply a variety of particle mixtures to a wide range of substrates and it allows to engineer the thin film depositions by manipulating the tuneable spray coating parameters. Spray coating is already established in many coating industries and currently researched for various emerging applications in nanotechnology including, organic [7] and hybrid (perovskite) [13] solar cells, OLEDs [14], electrodes (polymer [15]; metal nanoparticle [16]; carbon nanotubes [17]), etc.

The focus of our research was directed to deposit an ecofriendly, water-based nanoparticle ink into layers with desired thickness and morphology, by optimising the ultrasonic spray coating parameters. We studied the influence of the individual ultrasonic spray coating parameters as well as the influence of deposition temperature and thermal post treatment.

(b)

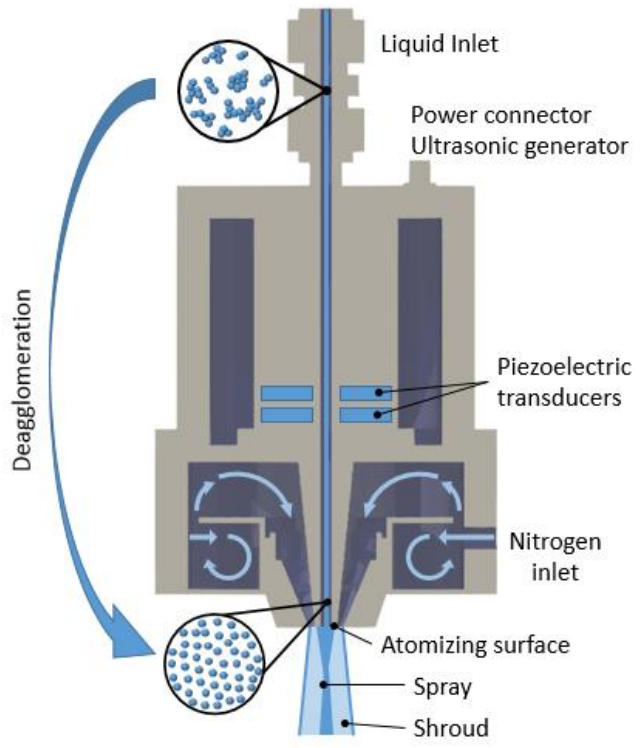

(a)

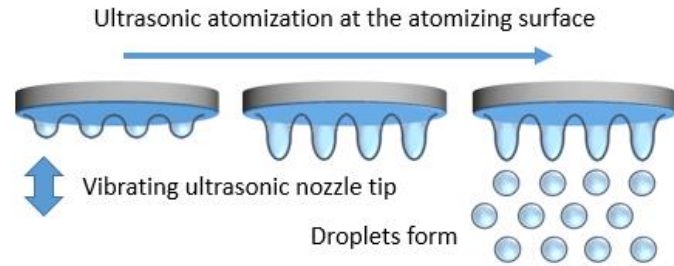

Figure 1 (a) Ultrasonic atomization principle at the atomizing surface (b) Cross-section of an ultrasonic spray nozzle and deagglomeration of nanoparticles

2 Materials and Methods In order to study the layer formation, packing density and uniformity under different conditions, pristine polystyrene NPs with an average diameter of $200 \mathrm{~nm}$ (dynamic light scattering:137.9 nm with a polydispersity index (PDI) of 0.130 ) were prepared -using the miniemulsion [18][19] technique and were used in this study as a proof-of-principle model system. Thus, $6.0 \mathrm{~g}$ of styrene, $250 \mathrm{mg}$ of the hydrophobic agent hexadecane and $100 \mathrm{mg}$ of the initiator V-59 were added to a surfactant solution containing $72 \mathrm{mg}$ of sodium dodecyl sulfate (SDS) and $24 \mathrm{~g}$ of water. After stirring for $1 \mathrm{~h}$ for preemulsification, the emulsion was subjected to ultrasonication (Branson 450W digital sonifier 1/2" tip) under ice cooling for $2 \mathrm{~min}$ at $90 \%$ amplitude. For polymerization, the miniemulsion was transferred to a round bottom flask and stirred overnight at $72^{\circ} \mathrm{C}$. The excess surfactant in the sample was removed by multiple washing steps using Milipore membrane tubes. The solid content as synthesized was $14.68 \%$.

The glass substrates were cleaned by sonication in soapy (Brandson GP) water solution for $30 \mathrm{~min}$, followed by sonication in distilled water and then in acetone for 5 and 10 min respectively. Finally they were put in hot isopropyl alcohol for 10 min and dried in air with a nitrogen gun.

A commercial ultrasonic spray coater (Model: ExactaCoat) from Sono-Tek was employed to print water-based polystyrene NPs diluted using water-ethanol solution under different conditions. This ultrasonic spray coater applies harmonic vibrations to pulverise the liquid into an aerosol. The vibrations create standing waves in the liquid at the atomizing surface of the nozzle (Figure $1 \mathrm{a}$ ). With enough power, cavitation occurs at the liquid gas interface resulting in an aerosol with a narrow droplet size distribution closely related to the capillary wavelength at the liquid surface. Frequency is the predominant factor determining the droplet size following from the Kelvin's equation for the capillary wavelength. Lang was able to empirically link the droplet diameter (D) to the ultrasound frequency (f) [20]:

$D=0,34 \times\left(\frac{8 \pi \sigma}{\rho f^{2}}\right)^{1 / a} \approx\left(\frac{\pi \sigma}{4 \rho f^{2}}\right)^{1 / 3 a}$

where $\sigma$ is the liquid surface tension and $\rho$ its density. The piezoelectric transducers of the nozzles used in this work had all an operational frequency of about $120 \mathrm{kHz}$. This results in an average mean droplet size of about $20 \mu \mathrm{m}$.

As the liquid is flowing through the spray head (Figure 1b), the NPs are exposed to the ultrasonic vibrations and a deagglomeration of the NPs takes place. Due to the relative low power and short time the liquid is exposed to the vibrations, the effect is limited to small aggregates so it is important to start with a high quality dispersion.

After the droplets are separated from the nozzle the nitrogen carrier gas directs the droplets towards the substrate. The precise control over the pressure of the carrier gas allows optimising the kinetic energy of the droplets upon impact on the substrate.

The surface structure and coverage was studied by an Axiovert 40 MAT optical microscope. The thickness and 
roughness of the spray coated layers were measured by a DektakXT stylus profiler from Bruker. To examine the layer formation and (nano-)morphological aspects of the layers a FEI Quanta 200F Field Emission Gun Scanning Electron Microscope (FEG-SEM) was used.

\section{Results and discussion}

Although the solvent to co-solvent ratio and NP-content were changed to some extent, the research mainly focused on how the change of the independent spray coating variables influenced the resulting layers.

First the ink formulation (water to co-solvent ratio and NP content) was optimised to influence the main indirect variable, the so-called coating efficiency, which depends on the effective chemistry between substrate and ink. When the ink reached the desired wetting properties, required to achieve thin and homogeneous layers, fully covering the substrate (Figure 2), the direct spray coater settings (flow rate, spray speed and temperature) were tuned to finalize with a thin and very smooth polystyrene layer.

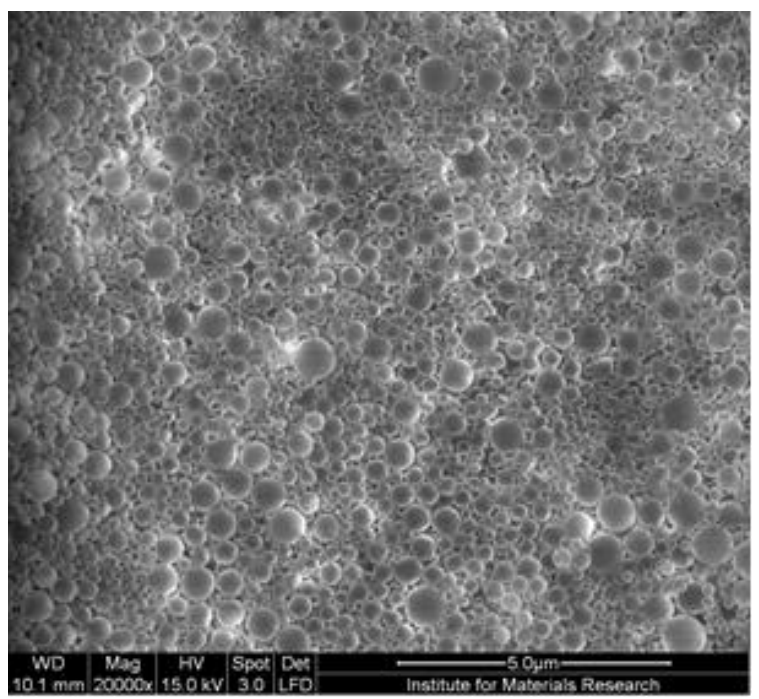

Figure 2 Optimized $10 \mu \mathrm{m}$ thick polystyrene nanoparticle layer

3.1 Initial layer formation Since the same inkformulation (1 wt $\%$ ) was used throughout most experiments, it was possible to observe the change in surface free energy during deposition. The initial droplets that hit the bare substrate face different wetting properties and had a higher particle mobility than the particles deposited on a covered substrate. Partly due to this higher mobility of the particles in the water based ink, the coffee ring effect could be observed. There are many ways to counter or minimalize this effect [21][22]. In our experiments the countermeasures were confined to the solvent to co-solvent ratio and temperature. In different experiments, the higher ethanol concentration appeared better to create thin nanoparticle layers as the fast dry-time locked the particles in place.
Although the surface tension is practically halved with the addition of $20 \mathrm{wt} \%$ of ethanol [23] and an initially low contact angle is observed, it rapidly retracts into large clusters with contact angles close to that of pure water [24]. To reach the desired wetting effect on our substrate we need more than $50 \mathrm{wt} \%$ of ethanol as previously observed for the spraycoating of polymer solar cells [25]. This dewetting-effect is more pronounced at lower temperatures. Concentrations of ethanol over $70 \mathrm{wt} \%$ however led to agglomeration of the particles in the ink.

When the coating efficiency of the ink is tuned, one can start the optimisation of the spray coating process. Vak et al. [26] divided the distance between airbrush nozzle and substrate in three different regions, which are "wet", "intermediate", and "dry". They concluded, as confirmed by Susanna et al. [27] and our experimental results, that the best linear control over layer formation was in the "intermediate zone". The great advantage of ultrasonic spray coating over airbrush is the independent control over the process variables like droplet size, the kinetic energy of the droplets and the volume of sprayed liquid. This allows to reach this desired "intermediate state" through different approaches.

In Table 1 the ranges of the tested spray coating parameters are displayed. Numerous experiments were conducted to search for the optimum for the different settings.

Table 1 Range of the tested spray coating parameters

\begin{tabular}{ll}
\hline Parameter & Tested setting range \\
\hline Flow rate & $0.05-1 \mathrm{~mL} / \mathrm{min}$ \\
Nozzle Speed & $1-50 \mathrm{~mm} / \mathrm{s}$ \\
Nozzle Power & $1.7-5 \mathrm{~W}$ \\
Co-solvent ratio $\mathbf{H}_{2} \mathbf{O} / \mathbf{C}_{2} \mathbf{H}_{5} \mathbf{O H}$ & $70 / 30-30 / 70[\%]$ \\
NP-content & $0.1-3 \mathrm{wt} \%$ \\
Temperature & $\mathrm{RT}-250{ }^{\circ} \mathrm{C}$ \\
\# layers & $1-50$ \\
\hline
\end{tabular}

Each parameter had the ability to change the layer morphology tremendously. The SEM images in Figure 3 show the impact a relative small change of one of the parameters has on the layer formation. The increase in substrate temperature above $70{ }^{\circ} \mathrm{C}$ increased the roughness significantly as the layer dried almost instantly. When increasing the nozzle velocity, one can tune the flowrate in order to deposit the same amount of material on a given area by raising it accordingly. The layer formation will change as more material is deposited in less time, further increase will eventually lead to the undesired "wet film" phenomenon. 
(a)

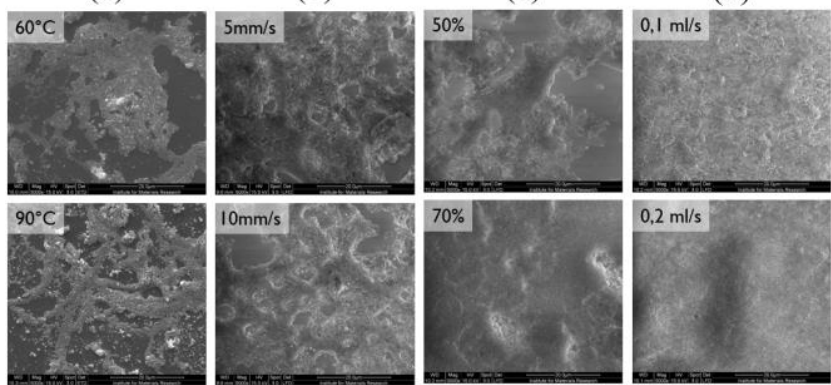

Figure 3 SEM images displaying the influence on the morphology for different parameter alterations. a) Temperature, b) Nozzle speed, c) ethanol/water ratio, and d) Flow rate

It can be inferred from these results that optimization of individual parameters is very crucial for obtaining optimal layers. Also, the choice of the solvents (vapor pressure) makes the process challenging as the ambient conditions (temperature/humidity) can play a decisive role. It is widely known that humidity plays an important role during the curing of waterborne coatings. The relative humidity affects the speed at which the water and coalescing solvents evaporate relative to each other.

3.2 Nanoparticle stacking in thick layers As a next step, the influence on the stacking of the NPs under different conditions was studied to see how the final layer morphology was formed. Different multilayer approaches to improve the coverage and density of the NP layer were compared to single layers.

After the substrate became partly covered, the mobility of the particles was reduced further due to the increased roughness and change in surface free energy. In this phase of the layer formation a higher particle mobility (i.e. lower ethanol concentration and/or temperature) was needed to reduce the roughness below the particle size.

3.3 Influence of temperature Finally, the effect of a thermal post-treatment was studied. Temperatures above the glass transition temperature ( $\mathrm{Tg}$ ), to increase adhesion to the coated surface and above the melting point (Mp), to create dense thin layers were used and their influence was studied.
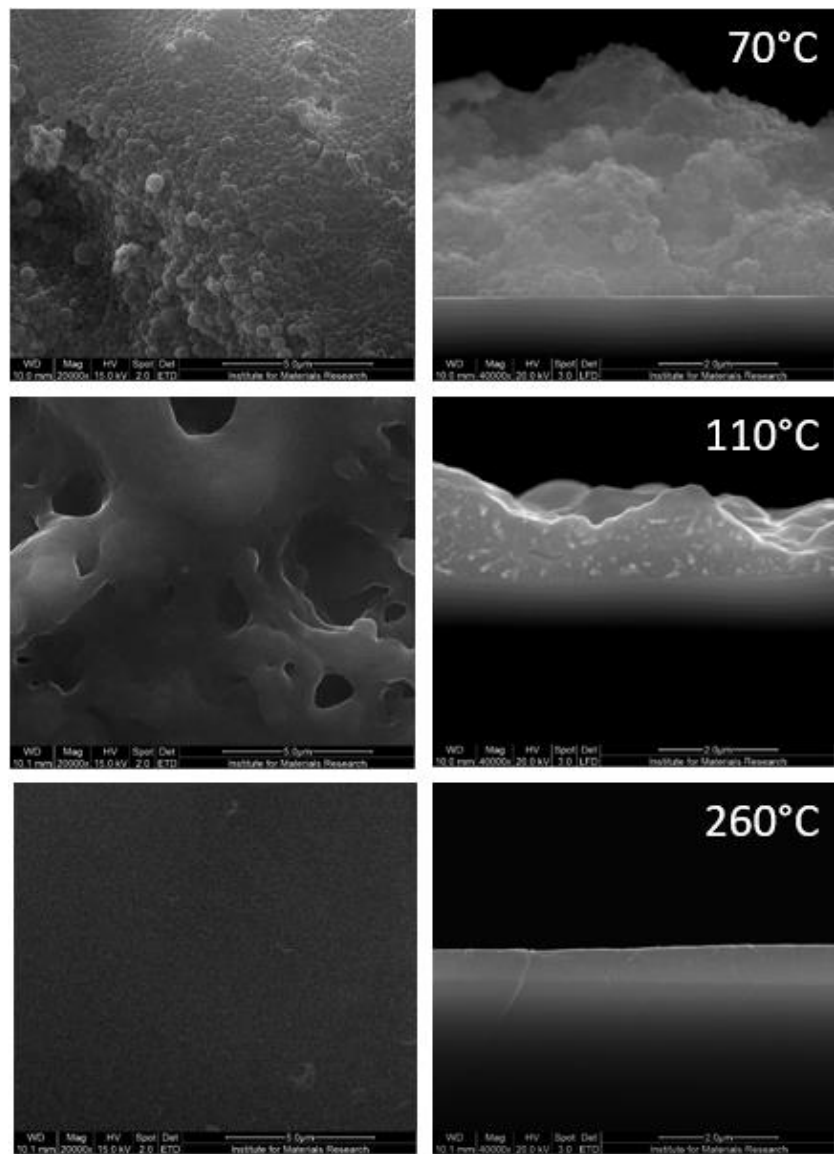

Figure 4 SEM images of layers during the annealing process. (Left) Top-view; scale bar is $5 \mu \mathrm{m}$ (Right) cross-section; scale bar is $2 \mu \mathrm{m}$.

As already discussed above, temperature is an important variable to influence the dry-time of the layer and the mobility of the NPs. As mentioned earlier, even the change in room temperature (RT) plays a significant role. Since we observe this behaviour during our experiments at RT we conducted further experiments at a minimum of $30{ }^{\circ} \mathrm{C}$ to reduce the effect. Temperature can partly compensate for the lack of the preferred volatile solvents to maximize liquid evaporation and reduce any potential particle aggregation associated with capillary forces during drying. Because there is no binder or other adhesive in our ink, the layer can easily be removed. Temperatures above the glass $\mathrm{Tg}\left(100{ }^{\circ} \mathrm{C}\right)[28]$ can be used to make the particles stick without particle deformation as can be observed in SEM. It is even possible to do this temperature treatment during the deposition process as the temperature of the substrate table can be ramped up to $250{ }^{\circ} \mathrm{C}$. Higher temperatures change the layer formation too much and are only possible as a post-treatment. With various temperature post-treatments above $\mathrm{Tg}$, mesoporous layers with a lower roughness could be achieved. A free surface is known to cause reductions in the Tg of thin polystyrene films [3]. Since the free surface area is orders of magnitude larger than for solid films, 
combined with the relative low molecular weight [4], a significant decrease in $\mathrm{Tg}$ was expected. In Figure 4, the change in morphology for different post-treatment temperatures can be observed.
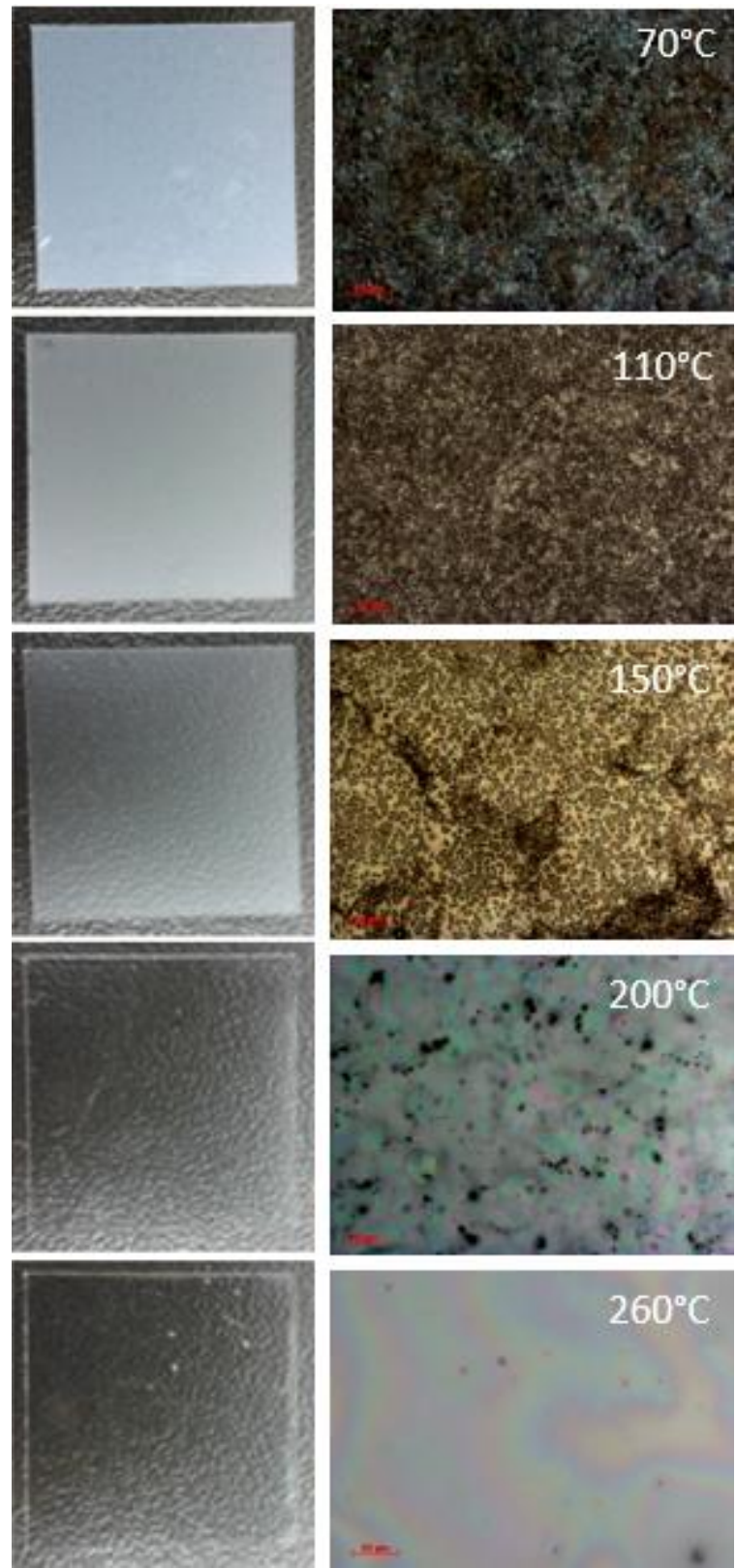

Figure 5 (Left) Digital photographs of the annealed layers on $18 \times 18 \mathrm{~mm}$ glass substrates, respectively (top to bottom), as deposited at $70{ }^{\circ} \mathrm{C}$ and after annealing for 5 min. at $110 ; 150 ; 200 ; 260{ }^{\circ} \mathrm{C}$. (right) Optical microscopy images from the change in morphology during annealing. (Scale bar is $20 \mu \mathrm{m}$ )

At $110{ }^{\circ} \mathrm{C}$, well above the $\mathrm{Tg}$, a mesoporous layer is formed. Due to thermal decomposition [29], the particles melted together at much lower temperatures than the melting temperature $\left(\sim 240{ }^{\circ} \mathrm{C}\right)$ [28] of the material. It formed into a dense layer at $150{ }^{\circ} \mathrm{C}$, which reduced even further in thickness and average roughness at higher temperatures. (Figure 5 left) The optical transparency increased as well at higher temperatures as can be observed in Figure 5 left. All aggregates broke up at $260{ }^{\circ} \mathrm{C}$ (Figure 5 right) and the most uniform dense layer was formed at this temperature (Figure 4). Higher temperatures result in dewetting of the molten polystyrene layer and eventually even in the formation of molten polystyrene droplets on the substrate.

4 Conclusion Water-ethanol-based polystyrene NPdispersions were spray coated on glass substrates under a wide range of different parameter conditions in order to study the layer formation and its final morphology. The ultrasonic spray coater allowed studying the influence of the different parameters separately and various approaches could lead to similar uniform NP-layers. The influence of temperature for deposition and post-treatment was studied and led to mesoporous and dense polystyrene layers. It was demonstrated that dense polystyrene layers are obtained using water based dispersions with low solid contents. The presented experimental results on the model system of polysterene NPs has therefore demonstrated that spray coating is a promising route towards a reliable, flexible and cost efficient fabrication method for printing nanosuspension dispersions.

Acknowledgements The authors would like to thank financial support from the Interreg project SolarFlare. A.E. is a FWO (Research Foundation Flanders) postdoctoral fellow. J.V.M.S., C.K.A. and A.C.G.L. are grateful to AUCANI and ProInt - USP for the scholarships.

\section{References}

P. Couvreur, "Nanoparticles in drug delivery: past, present and future.," Adv. Drug Deliv. Rev., vol. 65, no. 1, pp. 21-3, Jan. 2013.

T. Patel, J. Zhou, J. M. Piepmeier, and W. M. Saltzman, "Polymeric nanoparticles for drug delivery to the central nervous system.," Adv. Drug Deliv. Rev., vol. 64, no. 7, pp. 701-5, May 2012.

[3] J. S. Sharp and J. A. Forrest, "Free Surfaces Cause Reductions in the Glass Transition Temperature of Thin Polystyrene Films," Phys. Rev. Lett., vol. 91, no. 23, p. 235701, 2003.

H. Yin, D. Cangialosi, and A. Schönhals, "Glass transition and segmental dynamics in thin supported polystyrene films: The role of molecular weight and annealing," Thermochim. Acta, vol. 566, pp. 186-192, Aug. 2013.

A. Stapleton, B. Vaughan, B. Xue, E. Sesa, K. Burke, X. Zhou, G. Bryant, O. Werzer, A. Nelson, A. L. David Kilcoyne, L. Thomsen, E. Wanless, W. Belcher, and P. Dastoor, "A multilayered approach to polyfluorene water-based organic photovoltaics," Sol. Energy Mater. Sol. Cells, vol. 102, pp. 114-124, 2012. 
[6] M. Bag, T. S. Gehan, D. D. Algaier, F. Liu, G. Nagarjuna, P. M. Lahti, T. P. Russell, and D. Venkataraman, "Efficient Charge Transport in Assemblies of Surfactant-Stabilized Semiconducting Nanoparticles," Adv. Mater., vol. 25, no. 44, pp. 64116415,2013

[7] F. Aziz and A. F. Ismail, "Spray coating methods for polymer solar cells fabrication: A review," Mater. Sci. Semicond. Process., vol. 39, pp. 416-425, Nov. 2015.

[8] T. P. Bigioni, X.-M. Lin, T. T. Nguyen, E. I. Corwin, T. A. Witten, and H. M. Jaeger, "Kinetically driven self assembly of highly ordered nanoparticle monolayers.," Nat. Mater., vol. 5, no. 4, pp. 265-270, 2006.

[9] Y.-K. Hong, H. Kim, G. Lee, W. Kim, J.-I. Park, J. Cheon, and J.-Y. Koo, "Controlled two-dimensional distribution of nanoparticles by spin-coating method," Appl. Phys. Lett., vol. 80, no. 5, p. 844, 2002.

[10] H. Ringsdorf, B. Schlarb, and J. Venzmer, "Molecular Architecture and Function of Polymeric Oriented Systems: Models for the Study of Organization, Surface Recognition, and Dynamics of Biomembranes," Angew. Chem. Int., vol. 27, no. 1, pp. 113-158, 1988.

[11] X. Wu, I. Wyman, G. Zhang, J. Lin, Z. Liu, Y. Wang, and $\mathrm{H}$. $\mathrm{Hu}$, "Preparation of superamphiphobic polymerbased coatings via spray- and dip-coating strategies," Prog. Org. Coatings, Aug. 2015.

[12] J. Magura, A. Zeleňáková, V. Zeleňák, and M Kaňuchová, "Thiol-modified gold nanoparticles deposited on silica support using dip coating," Appl. Surf. Sci., vol. 315, pp. 392-399, Oct. 2014.

[13] A. T. Barrows, A. J. Pearson, C. K. Kwak, A. D. F. Dunbar, A. R. Buckley, and D. G. Lidzey, "Efficient planar heterojunction mixed-halide perovskite solar cells deposited via spray-deposition," Energy Environ. Sci., vol. 7, no. 9, pp. 2944-2950, 2014.

[14] K. Gilissen, J. Stryckers, P. Verstappen, J. Drijkoningen, G. H. L. Heintges, L. Lutsen, J. Manca, W. Maes, and W. Deferme, "Ultrasonic spray coating as deposition technique for the light-emitting layer in polymer LEDs," Org. Electron., vol. 20, pp. 31-35, May 2015.

[15] J. G. Tait, B. J. Worfolk, S. A. Maloney, T. C. Hauger, A. L. Elias, J. M. Buriak, and K. D. Harris, "Spray coated high-conductivity PEDOT:PSS transparent electrodes for stretchable and mechanically-robust organic solar cells," Sol. Energy Mater. Sol. Cells, vol. 110, pp. 98-106, Mar. 2013.

[16] C. Girotto, B. P. Rand, S. Steudel, J. Genoe, and P. Heremans, "Nanoparticle-based, spray-coated silver top contacts for efficient polymer solar cells," Org. Electron., vol. 10, no. 4, pp. 735-740, Jul. 2009.

[17] R. C. Tenent, T. M. Barnes, J. D. Bergeson, A. J. Ferguson, B. To, L. M. Gedvilas, M. J. Heben, and J. L. Blackburn, "UItrasmooth, large-area, high-uniformity, conductive transparent single-walled-carbon-nanotube films for photovoltaics produced by ultrasonic spraying," Adv. Mater., vol. 21, no. 31, pp. 3210-3216, 2009.

[18] K. Landfester, "Miniemulsion Polymerization and the Structure of Polymer and Hybrid Nanoparticles," Angew. Chemie Int. Ed., vol. 48, no. 25, pp. 4488-4507, 2009.

[19] A. Ethirajan and K. Landfester, "Functional Hybrid Materials with Polymer Nanoparticles as Templates," Chem. - A Eur. J., vol. 16, no. 31, pp. 9398-9412, 2010.

[20] R. J. Lang, "Ultrasonic Atomization of Liquids," $J$ Acoust. Soc. Am., vol. 34, no. 1, pp. 6-8, 1962.

[21] X. Shen, C. M. Ho, and T. S. Wong, "Minimal size of coffee ring structure," J. Phys. Chem. B, vol. 114, no. 16, pp. 5269-5274, 2010.

[22] D. Soltman and V. Subramanian, "Inkjet-printed line morphologies and temperature control of the coffee ring effect," Langmuir, vol. 24, no. 5, pp. 2224-2231, 2008.

[23] D. R. Lide, "CRC Handbook of Chemistry and Physics," p. 3485, 2003.

[24] C. Liu, E. Bonaccurso, and H.-J. Butt, "Evaporation of sessile water/ethanol drops in a controlled environment," Phys. Chem. Chem. Phys., vol. 10, no. 47, p. 7150, 2008.

[25] C. Girotto, D. Moia, B. P. Rand, and P. Heremans, "High-Performance Organic Solar Cells with SprayCoated Hole-Transport and Active Layers," Adv. Funct. Mater., vol. 21, no. 1, pp. 64-72, 2011.

[26] D. Vak, S.-S. Kim, J. Jo, S.-H. Oh, S.-I. Na, J. Kim, and D.-Y. Kim, "Fabrication of organic bulk heterojunction solar cells by a spray deposition method for low-cost power generation," Appl. Phys. Lett., vol. 91, no. 8, p. 081102, 2007.

[27] G. Susanna, L. Salamandra, T. M. Brown, A. Di Carlo, F. Brunetti, and A. Reale, "Airbrush spray-coating of polymer bulk-heterojunction solar cells," Sol. Energy Mater. Sol. Cells, vol. 95, no. 7, pp. 1775-1778, Jul. 2011.

[28] J. a. Sauer, Polymer Science and Technology, vol. 74, no. 2. 1985.

[29] S. L. Malhotra, J. Hesse, and L. P. Blanchard, "Thermal Decomposition of Polystyrene," Polymer (Guildf)., vol. 16, no. 2, pp. 81-93, 1975. 